\title{
Surgical management of pain
}

\author{
S J Rothemeyer, ${ }^{1}$ MB ChB, FCNeurosurgery (SA); J M N Enslin, ${ }^{2}$ BPhysT, MB ChB, FCNeurosurgery (SA), MMed (Neurosurg) \\ ${ }^{1}$ Department of Neurosurgery, Faculty of Health Sciences, Groote Schuur Hospital and University of Cape Town, South Africa \\ ${ }^{2}$ Division of Neurosurgery, Red Cross War Memorial Children's Hospital, and Constantiaberg Mediclinic, Cape Town, South Africa
}

Corresponding author: J M N Enslin (enslin@functionalneurosurgery.co.za)

Severe and intractable pain is one of the most difficult and challenging neurological conditions to deal with and to treat. The entity is not entirely well understood, and the afflicted patients often have significant concomitant neuropsychological problems that obscure the physical issue at hand. Physicians also do not fully understand what pain is. In a sense all pain is neural in origin. From a therapeutic perspective, pain is divided into visceral (dull and poorly localised owing to enteric sensory receptors) and somatosensory (more discreet and localised - often owing to nociceptors being stimulated) pain. It is detected by nociceptors, i.e. sensory reseptors with the ability to interpret and transmit noxious stimuli. Treatment options include medication, physical therapy and psychotherapy. The availability of sophisticated new medication, such as pregabalin, augments the medical arm of therapy. If these therapies fail, and with a thorough multidisciplinary approach involving carefully screened cases, surgery may form part of the management. Generally, surgical pain management is divided into neuromodulative (enhancing physiological control of the pain system) and neurodestructive (lesioning and destroying the defined pain generator in the central nervous system) surgery. Complex pain management should not focus on cure as the only outcome. Patients often experience pain for years before considering surgery; it would therefore be unwise to expect an immediate cure. Careful psychological support and evaluation is of the utmost importance. This article gives an overview of the neurosurgical management of pain.

S Afr Med J 2016;106(9):858-860. DOI:10.7196/SAMJ.2016.v106i9.11366

To understand the management of pain, it is important to focus on some basic aspects of pain physiology. A detailed explanation of this topic is, however, beyond the scope of this article.

Neuropathic pain is described as 'pain arising as a direct consequence of a lesion or disease affecting the somatosensory system. ${ }^{[1]}$ The Kyoto Protocol of the International Association for the Study of Pain (IASP) defined pain as follows: 'Pain is an unpleasant sensory and emotional experience associated with actual or potential tissue damage or described in terms of such damage. ${ }^{[1]}$ The pain can be peripheral or central (depending on whether the peripheral nervous system or central nervous system is involved). ${ }^{[1]}$ In 1986, the World Health Organization (WHO) first published its 'pain ladder ${ }^{\text {'2] }}$ approach to the management of pain. Even in severe or complex pain management algorithms, these steps are still followed. The first step includes the use of paracetamol or a nonsteroidal anti-inflammatory drug (NSAID). If these do not relieve the pain, then adjunctive drugs are added, such as anticonvulsants (gabapentin or pregabalin) or a tricyclic antidepressant (amitriptyline). Further severe pain requires the addition of an opiate (e.g. morphine). ${ }^{[2]}$ Patients with pain that does not respond to this ladder approach, should be referred to a specialist pain management centre. The multidisciplinary team should ideally consist of an anaesthetist with a special interest in pain management, social worker, psychologist, psychiatrist, physiotherapist, occupational therapist and functional neurosurgeon. ${ }^{[3]}$ Various other ancillary therapeutic methods, such as musical therapy and play therapy, are often also involved and may aid in managing complex pain.

\section{Pathophysiology}

Pain is defined as a nociceptive and emotional response to a lesion or a disease that affects the somatosensory system. ${ }^{[1]}$ This system comprises the brain and the areas in the brain that are intricately involved with our sensory experience of our surroundings - the thalamus and its nuclei that act as major relay centres for the information transferred to the sensory cortex from all the receptive organs in our bodies. This information is transmitted to the thalamus and the rest of the central nervous system via long white matter tracts in the spinal column and brainstem (spinothalamic and reticulothalamic tract). The peripheral nerves act as afferent pathways for neural messages from the nociceptive receptors to the spinal cord, and the efferent pathways that relay the messages to react to the stimulus. ${ }^{[4]}$ Fig. 1 is a schematic illustration of a cross-sectional cut through the spinal cord. ${ }^{[4]}$ In this article, the reader is referred back to this illustration to understand the surgical techniques used to manage pain.

Pain is caused by nociceptor activation by noxious stimuli (chemical, mechanical or temperature) that initiate a cascade of

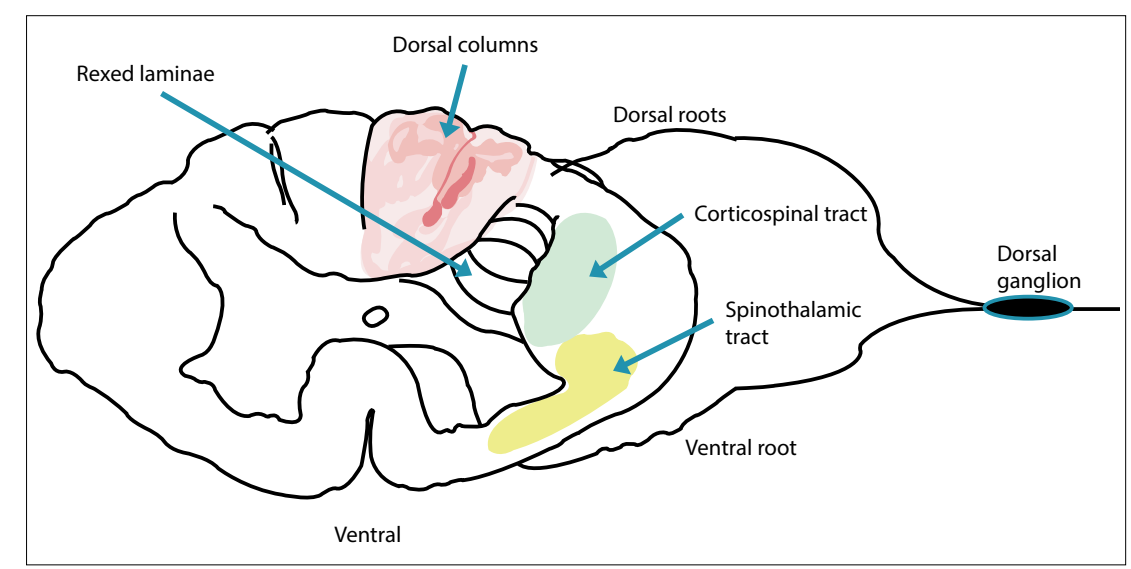

Fig. 1. Sketch of an axial cut through the spinal cord and nerve roots, illustrating the ganglion and the different white and gray matter sections involved in pain surgery. ${ }^{[4]}$ 
neurotransmitter substances, which lead to an electrical potential change in nerve fibres. Pain is transmitted via thick, myelinated A ( $\beta$ and $\delta$ )-fibres, as well as thin, unmyelinated C-fibres. Pain can therefore be 'gated' by using this two-fibre process to one's advantage. There are certain therapies, such as transcutaneous electrical nerve stimulation (TENS) and other physiotherapeutic activities, which cause stimulation of the A-fibres, thereby blocking the slower $\mathrm{C}$-fibres and modulating pain.

Central neural injury involves injury to any part of the central nervous system from the dorsal root entry zone (DREZ) second-order neurons up to the sensory cortex, i.e. somewhere along the path of the spinoreticulothalamic system. This was historically called 'central pain'. In cases of such pain, it has been shown that thalamic neurons have an abnormal, spontaneous high-frequency discharge pattern, firing in bursts. This may be the reason why deep brain stimulation (DBS) of the thalamus and motor cortex stimulation are effective to treat central pain. ${ }^{[5,6]}$

Neural injury can be traumatic (blunt, such as crushing; or sharp, such as cutting), avulsive (e.g. brachial plexus injuries); chemical; electrical; or thermal. Regardless of the mechanism, damaged primary afferent neurons undergo similar changes. The injured nerves are sensitised and discharge spontaneously. They may become hypersensitive to noradrenaline, which contributes to the complex regional pain syndrome. As the damaged nerve attempts to regenerate, the regenerating sprouts may produce ectopic discharges. Demyelinated nerve fibres have 'ephaptic' electrical transmission, where adjacent fibres activate each other. Isolated demyelinated axons can generate reflected neural impulses, which move in directions opposite to normal electrical flow; this may cause the 'buzzing' feeling some people experience with pain. ${ }^{[4]}$

Peripheral nerve injuries can affect function up to the point of termination in the dorsal horn (Fig. 1) - these abnormal primary neurons sprout and colonise new areas within the dorsal horn. Injured afferent neurons produce different neuropeptides to the ones they normally produce. Injured dorsal root neurons discharge at higher frequencies and more spontaneously than normally.

\section{Surgical techniques}

The physician should ensure that treatable causes of the pain have been carefully identified and excluded. Examples include temporomandibular joint disorder, dental disease, malocclusion causing facial pain (incorrectly labelled as trigeminal neuralgia), nerve compression syndromes (carpal tunnel syndrome is the most common), and radicular arm or leg pain of spinal origin (e.g. degenerative cervical spondylotic changes causing burning arm or hand pain) Neuropathic pain should be considered a diagnosis of exclusion, arrived at after diligent, careful screening of a patient.

Once pain is confirmed to be refractory to all medical therapy and adjuncts such as music therapy, physiotherapy, and cognitive behavioural therapy, the pain team should consider surgery. The techniques available in the surgical management of pain are summarised in Table $1 .^{[5-12]}$

Surgery can help in the management of pain in the following ways:

- by modulating neural function: ${ }^{[5-8]}$ TENS, spinal cord stimulation (SCS), motor cortex stimulation (MCS), and DBS

\section{Table 1. Possible surgical therapies for managing complex pain}

\begin{tabular}{lll}
\hline Lesional procedures & Neuromodulation & Other \\
\hline - Dorsal root entry zone & - Spinal cord stimulation & - Intrathecal medical therapy \\
lesioning & - Peripheral nerve & Microvascular \\
- Dorsal rhizotomy & stimulation & \\
- Dorsal root ganglionectomy & - Motor cortex stimulation & \\
- Rhizolysis of nerve ganglion & - Cranial nerve stimulation \\
- Neurectomy & - Deep brain stimulation & \\
- Stereotactic radiosurgery & \\
- Percutaneous & \\
radiofrequency gangliolysis & \\
- Mechanical balloon & \\
gangliolysis & \\
- Image-guided tractotomy & \\
and nucleotomy & \\
- Cordotomy & \\
- Midline myelotomy &
\end{tabular}

- by destroying neural function: ${ }^{[12,13]}$ avulsing the afflicted nerve(s), e.g. DREZ lesioning, myelotomy, tractotomy, cingulotomy, or thalamotomy, in the hope of removing the short-circuited, dysfunctional paingenerating nerves from the normal neural system, or interrupting the feedback pathway to the brain.

A single injured nerve can be freed up, and the neuroma scar resected. Multiple peripheral nerves may be treated similarly, but rather than operating and injuring several functional nerves, multiple nerves are often treated non-invasively with electrical modulation, using TENS or SCS, located either at the corresponding spinal segment or the DREZ. Similarly, craniofacial pain syndromes, such as trigeminal neuralgia, are successfully treated by moving the pulsating artery that compresses the mandibular nerve in the cerebellopontine angle with microvascular decompression or, in the absence of an offending artery, performing a blockade of the trigeminal ganglion with balloon compression or glycerol rhizolysis. ${ }^{[10,11]}$

If the injury occurs distal to the dorsal root ganglion (DRG) (Fig. 1) - a major relay for sensory/pain input - and it is intact, then reversible modulation more central to the DRG, such as SCS or intrathecal morphine therapy, is possible. ${ }^{[7,9]}$ SCS activates the inhibitory effect of large primary afferent neurons in the dorsal columns, damping the incoming pain signal. If the DRG is injured, it causes corresponding injury to the dorsal columns and renders them unusable to modulation by SCS; ${ }^{[5]}$ any modulation target is then selected 'deeper' to this level, e.g. the contralateral thalamus or motor cortex.

DREZ lesioning is chosen for plexus or root lesions where the root(s) or plexus has been avulsed from the spinal cord and the DREZ relay cells are hyperactive, which contributes to the pain. ${ }^{[13]}$ DREZotomy is also useful in managing spasticity. ${ }^{[13,14]}$ More central, destructive procedures such as cordotomy and midline myelotomy of the spinal cord are reserved for patients in whom sensory loss is already present (e.g. after complete spinal cord injury) and who experience severe autonomic abnormalities or spasticity.

In cases of spinal cord injury, pain is frequently a confounding factor. The physician must always rule out pain caused by ongoing mechanical instability (bony, ligamentous or both). Such pain could be classified as: radicular, segmental (at the level of the injured cord), infrasegmental, or visceral. DREZ lesioning is offered if the injury involves the dorsal columns. If these are intact, SCS is 
suggested. It is important to be sure that the underlying instability or mechanical compression has been dealt with before other therapies are considered. ${ }^{[9]}$

Cerebral causes of pain are rare, but do occur. Peripheral techniques, such as DREZ lesioning, will not be effective in these cases. A classic example is thalamic pain caused by stroke (DejerineRoussy syndrome), where MCS (sited over the opposite motor cortex) is offered. It is thought to inhibit overactive relay neurons in the thalamus. ${ }^{[5]}$

Long-standing, severe pain is often associated with comorbid disease and spasticity. The comorbidities should be treated aggressively and therapy for spasticity, such as DREZ lesioning or intrathecal baclofen therapy, can be used with great success to improve the quality of life and improve function. ${ }^{[14]}$ It is important to reiterate at this stage that the patient should be treated - not only his/her pain.

\section{Conclusion}

Neuropathic pain is complex and difficult to diagnose and treat. A multidisciplinary team, with expert management and decisionmaking, is key. Surgical treatment is one of the last resorts after maximal dose medical and other therapies have failed, and should be tailored to the type and anatomical level of neural injury. Surgery may be declined to patients who have comorbid psychosis, refractory depression or other conditions. Be aware that patients with chronic pain often self-medicate with elaborate regimens and may be using medications affecting coagulation (aspirin, NSAIDs, and herbal or homeopathic medication). Such self-medication must be screened out before suggesting any form of surgery, as bleeding in the central nervous system could have devastating effects.

Modulation is preferable to ablation, as the former is reversible. However, the surgical technique should be individualised to every case. Time and thought should be given to getting to know the patient and their social and psychological circumstances. Chronic pain management should not be treated in the 'stampede' clinics that mostly fill our hospital passages. A caring and unhurried approach is needed with the aid of a multidisciplinary team.

1. Loeser JD, Treede RD. The Kyoto protocol of IASP Basic Pain Terminology. Pain 2008;137(3):473-477. DOI:10.1016/j.pain.2008.04.025

2. World Health Organization.WHO’s Pain Relief Ladder. www.who.int/cancer/palliative/painladder/en/ (accessed 4 August 2016).

The British Pain Society. Guidelines for Pain Management Programmes for Adults. London: Churchill House, 2013.

Ingram S. Physiologic anatomy of nociception. In: Burchiel K, ed. Surgical Management of Pain. New York: Thieme, 2015:3-11.

. Sindou M, Maarrawi J, Mertens P. Motor cortex stimulation. In: Burchiel K, ed. Surgical Management of Pain. New York: Thieme, 2015:366-379.

Kalia SK, Hamani C, Rezai A, Lozano AM. Deep brain stimulation for chronic pain. In: Burchiel K, ed. Surgical Management of Pain. New York: Thieme, 2015:380-392.

7. Linderoth B, Meyerson BA. Spinal cord stimulation: Mechanisms of action. In: Burchiel K, ed. Surgical Linderoth B, Meyerson BA. Spinal cord stimulation: Mech
Management of Pain. New York: Thieme, 2015:319-333.

Management of Pain. New York: Thieme, 2015:319-333.
Harsh V, Viswanathan A. Peripheral nerve stimulation. In: Burchiel K, ed. Surgical Management of Harsh V, Viswanathan A. Peripheral ner
Pain. New York: Thieme, 2015:349-353.

9. Tavanaiepour D, Levy RM. An overview of the rational use of intrathecal analgesic therapies. In Burchiel K, ed. Surgical Management of Pain. New York: Thieme, 2015:393-407.

10. Burchiel K. Microvascular decompression for trigeminal neuralgia. In: Burchiel K, ed. Surgical Management of Pain. New York: Thieme, 2015:425-433.

1. Luther N, Kondziolka D, Lunsford LD. Percutaneous retrogasserian glycerol rhizolysis. In: Burchiel K, ed. Surgical Management of Pain. New York: Thieme, 2015:470-475.

12. Cleary DR, Cetas JS. Overview of destructive neurosurgical procedures for pain. In: Burchiel K, ed. Surgical Management of Pain. New York: Thieme, 2015:521-528.

13. Sindou M. Dorsal root entry zone lesions. In: Burchiel K, ed. Surgical Management of Pain. New York: Thieme, 2015:576-594.

14. Enslin JMN, Fieggen AG. Surgical management of spasticity. S Afr Med J 2016;106(8):753-756. DOI:10.7196/SAMJ.2016.v106i8.11225 